\title{
Hypoxia index precisely covers the roles of FHR deceleration and bradycardia in fetal monitoring
}

\author{
Kazuo Maeda* \\ Obstetrics \& Gynecology, Tottori university Medical School, Yonago, Japan
}

\begin{abstract}
Hypoxia Index (HI), the sum of deceleration durations (min) divided by the lowest FHR (bpm) and multiplied by 100 was reliable to estimate fetal outcome instead of early, late and variable FHR decelerations in FHR monitoring in the labor.
\end{abstract}

\section{Introduction}

The fetal outcome was uncertain in fetal monitoring by pattern classification, because fetal outcome after 2-3 repetition of late decelerations (LD ) was favorable, while $50 \mathrm{~min}$ LD repetition resulted severe fetal and neonatal asphyxia, associating the loss of FHR variability and infantile brain damage, where the threshold to develop ominous outcome was unknown. The threshold of severe variable decelerations was also vague. The author thought it is necessary to create reliable evaluation of FHR decelerations .

\section{Methods}

As fetal bradycardia is caused by the excitation of parasympathetic center with hypoxia, while there was no bradycardia when experimental animal was anesthetized [1], and apneic bradycardia recovered to normal FHR after infusion of oxygenated blood to anencephalic neonate [2], while there was fetal brain damage followed by cerebral palsy after the loss of FHR variability, thus, the sign of fetal brain damage is the loss of variability, but not the bradycardia or deceleration, namely, they will be only the reaction to environmental hypoxia. As short duration of low $\mathrm{PaO} 2$ in 2-3 LDs does not affect the brain, while the brain is damaged after the Iong exposure to hypoxia appeared in the sum of LD durations in highly repeated decelerations, where the hypoxic intensity will be shown by the inversion of nadir FHR, that is the lowest bradycardia. Therefore, the sum of deceleration durations were divided by the nadir of decelerations in the hypoxia index in the evaluation of fetal outcome, particularly in cases of the loss of variability followed by fetal brain damage or cerebral palsy.

\section{Results}

The Apgar score was 9 after 3 connected LDs with $45 \mathrm{sec}$ lag time was 9, where the hypoxia index (HI) was 6 and outcome was normal, while hypoxia index was 25 and 26 in two fetuses, where one was fetal asphyxia associated with the loss of variability and cerebral palsy, and another one of $50 \mathrm{~min} \mathrm{LD}$ and the loss of variability was Apgar 3, followed by the death due to infantile brain hemorrhage, while hypoxia index was 20-24 in abnormal FHR cases but associated neither loss of variability, nor cerebral palsy. Thus, the threshold neither to develop the loss of variability nor cerebral palsy is 20-24 [3], and the HI will be 20 in the decision of early delivery.

\section{Discussion}

The hypoxia index and the decision of early delivery will be adopted also to other decelerations including early and variable decelerations, and also the continuous fetal bradycardia, where the pattern classification of decelerations is discarded, instead numeric hypoxia index will take the place to predict the brain damage and cerebral palsy in fetal monitoring. Additional necessary analyses will be sinusoidal heart rate and that of A/B ratio [4]. The hypoxic-ischemic encephalopathy after the continuous fetal bradycardia will be more precisely predicted by the application of hypoxia index than the present state. The minutes after the onset of bradycardia is measured instead of the sum of deceleration duration, and the lowest FHR is the nadir of FHR in the continuous bradycardia.

\section{Conclusion}

The hypoxia index is useful not only in the evaluation of late deceleration, but also it is applied in all decelerations in the pattern classifications. Therefore, vague FHR pattern classification will be useless by the application of the hypoxia index, which will be particularly useful in computerized fetal heart rate monitoring.

\section{References}

1. Umezawa $\mathbf{J}$ (1975) Studies on the relation between heart rate and $\mathrm{PaO}_{2}$ in hypoxic rabbit: a comparative study for fetal heart rate change in labor. Acta Obstet Gynecol Jpn 28: 1203-1212.

2. Maeda K, Kimura S, Nakano H, et al. (1969) Studies on fetal pathophysilogy. Acto Obstet Gynecol 8; 877886.

Correspondence to: Kazuo Maeda, Honorary Professor, Department of Obstetrics \& Gynecology, Tottori university Medical School, Yonago, Japan, E-mail: maedak@mocha.ocn.ne.jp

Received: October 16, 2017; Accepted: October 31, 2017; Published: November 04, 2017 
3. Maeda K (2014) Modalities of fetal evaluation to detect fetal compromise prior to the development of significant neurological damage. J Obstet Gynaecol Re 40: 2089-2094. [Crossref]
4. Maeda K, Iwabe T, Yoshida S, Ito T, Minagawa Y, et al. (2009) Detailed multigrade evaluation of fetal disorders with the quantified actocardiogram. J Perinat Med 37: 392-396. [Crossref]

Copyright: (C2017 Maeda K. This is an open-access article distributed under the terms of the Creative Commons Attribution License, which permits unrestricted use, distribution, and reproduction in any medium, provided the original author and source are credited. 